\title{
Total Synthesis of Sespenine
}
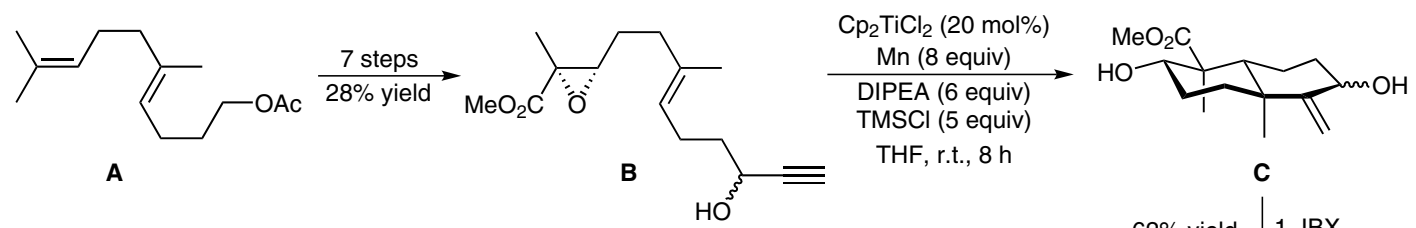

Synthesis of Natural

Products and

Potential Drugs

\section{Key words}

sespenine

indole

sesquiterpenoids

aza-Prins recation

Friedel-Crafts

reaction

radical cascades

Nysted olefination
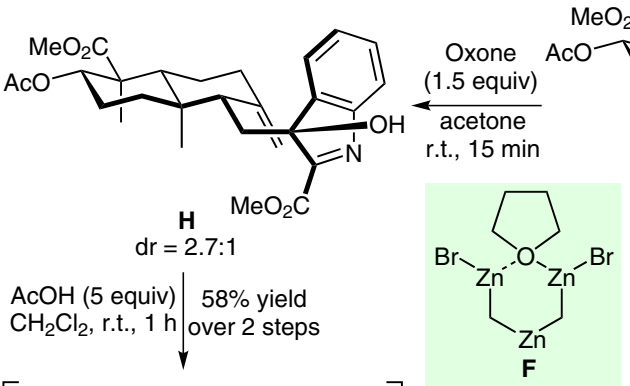

$\mathrm{HF}$, r.t., $8 \mathrm{~h}$

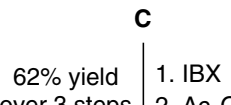

$\mathrm{AcOH}(5$ equiv) $58 \%$ yield $\mathrm{CH}_{2} \mathrm{Cl}_{2}$, r.t., $1 \mathrm{~h}$ over 2 steps
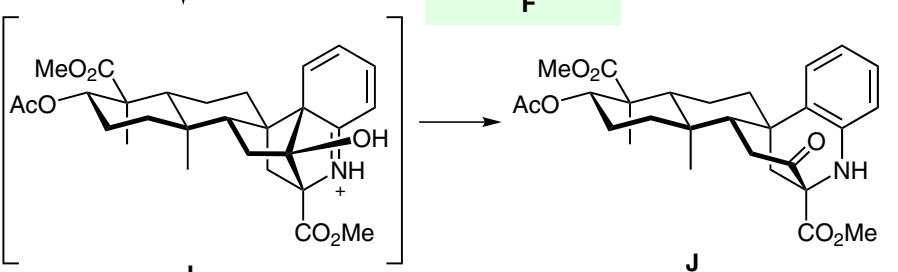

$\mathrm{MeCN}, 0^{\circ} \mathrm{C}$ over 3 steps $2 . \mathrm{Ac}_{2} \mathrm{O}, \mathrm{Et}_{3} \mathrm{~N}$

SYNFACT,
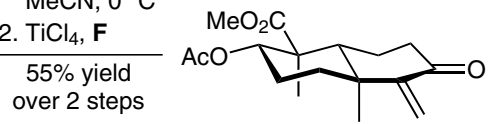
over 2 steps

D

G<smiles>CC(=O)c1cc2ccccc2[nH]1</smiles>

2 steps

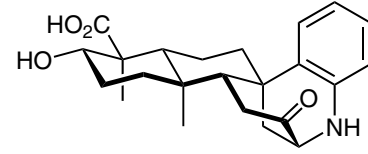

Sespenine

Significance: Sespenine is a rare and architecturally complex indole sesquiterpenoid that was isolated from an endophyte in 2011. Biosynthetically, sespenine may be formed from a structurally simpler indole precursor by oxidation of the indole $\mathrm{C} 3$ position followed by a cationic cascade involving an aza-Prins/Friedel-Crafts reaction and a subsequent fragmentation. Ang Li and co-workers now report the first total synthesis of sespenine. Their strategy relies on a titanium-mediated radical cascade for quick access to a key intermediate and an elegant implementation of the cationic cascade described above.
Comment: The synthesis commences with acetate $\mathbf{A}$, which is converted into $\alpha, \beta$-epoxy ester $\mathbf{B}$ in seven steps. Titanium(III)-mediated radical cyclization of $\mathbf{B}$ furnishes allylic alcohol $\mathbf{C}$, which is oxidized and protected to give enone $\mathbf{D}$. Key intermediate $\mathbf{G}$ is prepared via 1,4-addition of indole $\mathbf{E}$ and Nysted olefination using reagent $\mathbf{F}$. Oxidation of $\mathbf{G}$ with Oxone affords $\mathbf{H}$ as a 2.7:1 mixture of epimers. The major and desired product undergoes the crucial sequence of aza-Prins/FriedelCrafts reaction and fragmentation to give $\mathbf{J}$, presumably via the intermediacy of $\mathbf{I}$. Two additional steps then complete the synthesis of sespenine. 